\title{
BMJ
}

\section{Maintenance treatment with quetiapine versus discontinuation after one year of treatment in patients with remitted first episode psychosis: randomised controlled trial}

\author{
Eric Y H Chen, professor, ${ }^{1}$ Christy L M Hui, research fellow, ${ }^{1}$ May M L Lam, clinical assistant professor, ${ }^{1}$ Cindy P \\ Y Chiu, clinical assistant professor, ${ }^{1}$ C W Law, associate consultant, ${ }^{2}$ Dicky W S Chung, consultant, ${ }^{3}$ Steve Tso, \\ associate consultant, ${ }^{4}$ Edwin P F Pang, associate consultant, ${ }^{3} \mathrm{~K}$ T Chan, medical officer, ${ }^{4}{ }^{Y}$ C Wong, associate \\ consultant, ${ }^{3}$ Flora Y M Mo, associate consultant, ${ }^{3}$ Kathy P M Chan, associate consultant,, ${ }^{5}$ J Yao, associate \\ professor, ${ }^{6}$ S F Hung, consultant, ${ }^{5}$ William G Honer, professor ${ }^{7}$
}

\section{Department of Psychiatry, University of Hong Kong, Hong Kong \\ ${ }^{2}$ Department of Psychiatry, Queen Mary Hospital, Hong Kong \\ ${ }^{3}$ Department of Psychiatry, Tai Po Hospital, Tai Po, Hong Kong \\ ${ }^{4}$ Department of Psychiatry, Castle Peak Hospital, Tuen Mun, Hong Kong \\ ${ }^{5}$ Department of Psychiatry, Kwai Chung Hospital, Kwai Chung, Hong Kong \\ ${ }^{6}$ Clinical Trials Centre, University of Hong Kong, Queen Mary Hospital, Hong Kong \\ ${ }^{7}$ University of British Columbia, Centre for Complex Disorders, BCMHARI, A3-127, Vancouver, BC, Canada \\ Correspondence to: EY H Chen eyhchen@hku.hk}

Cite this as: BMJ 2010;341:C4024 doi:10.1136/bmj.c4024

\section{ABSTRACT}

Objective To study rates of relapse in remitted patients with first episode psychosis who either continued or discontinued antipsychotic drugs after at least one year of maintenance treatment.

Design 12 month randomised, double blind, placebo controlled trial.

Setting Early psychosis outpatient clinics in Hong Kong. Participants 178 patients with first episode psychosis who had received at least one year of antipsychotic drug treatment between September 2003 and July 2006 and had no positive symptoms of psychosis.

Interventions Patients received either maintenance treatment with quetiapine $(400 \mathrm{mg} /$ day) or placebo and were followed up for the next 12 months or until a relapse occurred.

Main outcome measure Relapse assessed monthly and defined as re-emergence of psychotic symptoms (delusions, conceptual disorganisation, hallucinations, suspiciousness, and unusual thought content) according to predefined thresholds.

Results 178 patients were randomised (89 to quetiapine and 89 to placebo). The Kaplan-Meier estimate of the risk of relapse at 12 months was $41 \%$ ( $95 \%$ confidence interval $29 \%$ to $53 \%$ ) for the quetiapine group and $79 \%$ (68\% to $90 \%$ ) for the placebo group ( $\mathrm{P}<0.001)$. Although quetiapine was generally well tolerated, the rate of discontinuation due to adverse or serious adverse events was greater in the quetiapine group $(18 \% ; 16 / 89)$ than in the placebo group (8\%; 7/89) (relative risk 2.29, $95 \%$ confidence interval 0.99 to $5.28 ; X^{2}=3.20, d f=1 ; P=0.07$ ).

Conclusion In a group of asymptomatic patients with first episode psychosis and at least one year of previous antipsychotic drug treatment, maintenance treatment with quetiapine compared with placebo resulted in a substantially lower rate of relapse during the following year.

Trial registration Clinical trials NCT00334035.

\section{INTRODUCTION}

Prevention of relapse is an important target in the treatment of schizophrenia and related psychotic conditions. ${ }^{1}$ In first episode psychosis, more than $80 \%$ of patients treated achieve a full response in psychotic symptoms, ${ }^{2}$ yet as many as $80 \%$ have a relapse within five years. ${ }^{3}$ Relapse compromises the outcome of psychotic disorders and is associated with substantial risks and costs. ${ }^{4}$ With each relapse, the response of symptoms to treatment seems to take approximately $50 \%$ longer and is increasingly difficult to reestablish. ${ }^{5}$ Prevention of relapse is particularly important in the early course of illness, when symptoms and disabilities may be less entrenched. Strategies to improve adherence and minimise early relapses are major focuses in treatment programmes and clinical research. ${ }^{1}$ For chronic psychotic illness, maintenance antipsychotic drug treatment is efficacious for preventing relapse. The rate of relapse after discontinuation of drug treatment in chronic schizophrenia is approximately $53 \%$ compared with $16 \%$ for patients maintained on antipsychotic drugs. ${ }^{6}$ Atypical antipsychotic drugs may have some benefits compared with typical antipsychotics in preventing relapse in chronic illness. ${ }^{78}$ The biological mechanism underlying relapse is not fully understood; a hyperdopaminergic state resulting from dysregulation and endogenous sensitisation of the central meso-limbic and meso-cortical dopamine systems have been proposed. ${ }^{9}$

For patients with first episode psychosis, the few randomised placebo controlled trials of relapse prevention were carried out in patients soon after discharge from hospital. ${ }^{10-12}$ Kane et al randomised 30 patients with at least four weeks of stable remission after admission to hospital (17 placebo $v 13$ fluphenazine) and found that $41 \%$ in the placebo group relapsed (defined as substantial clinical deterioration with marked social impairment) compared with none in the maintenance group. ${ }^{11}$ Crow et al randomised 120 patients within one month of discharge (66 on placebo $v 54$ on 
antipsychotics) and found that $62 \%$ of the placebo group and $46 \%$ of the maintenance group relapsed (resumption of drug or readmission to hospital) over the next 24 months. ${ }^{10}$ Hogarty et al studied 75 patients with two months of antipsychotic treatment after discharge from hospital and found that relapse (unequivocal clinical deterioration or imminent readmission) occurred in $64 \%$ of the placebo group and $43 \%$ of the maintenance group. ${ }^{12}$

Clinical guidelines recommend maintenance drug treatment for the first year after the onset of illness, but no consensus exists regarding longer periods of maintenance treatment. ${ }^{13-16}$ Some authors estimated that up to a quarter of patients with first episode psychosis may not need long term maintenance treatment, ${ }^{17}$ whereas others question whether it should ever be discontinued. ${ }^{1618}$ Two recent studies investigated first episode patients with somewhat longer periods of remission. Gitlin et al randomised 108 patients previously treated with depot fluphenazine for one year to placebo and maintenance groups (54 each) for three months, followed by a crossover of treatment in the next three months, then open label discontinuation. ${ }^{19}$ Exacerbation was defined as a recurrence of mild psychotic symptoms (a 2 point change, to a score $>3$, on any of three brief psychiatric rating scale psychosis items). Relapse was defined as a recurrence of severe psychotic symptoms (a rating of 6 or 7 on any of three brief psychiatric rating scale psychosis items). Unfortunately, interpretation of the effect of drug treatment was complicated by persistence of the depot fluphenazine in the placebo group. After open label discontinuation, 78\% had an exacerbation or relapse in the next 12 months. However, without randomisation and blinding, the effects of participants' and raters' expectations on the assessments remain uncertain. In another study of drug discontinuation, Wunderink et al randomised 128 patients who were in remission for six months $(65$ open withdrawal, 63 open continuation of drug). ${ }^{20}$ The study did not involve a placebo control. Relapse (clinical deterioration with one or more score in the severe range of 5 or more in the positive and negative syndrome scale positive subscale ${ }^{21}$ ) was more frequent in the discontinuation group (43\%) than in the maintenance treatment group (21\%).

In the context of the limited available evidence, discontinuation of drug treatment is perceived as a viable option for patients with first episode psychosis after at least one year of antipsychotic drug treatment without relapse. ${ }^{322}$ Longer term maintenance treatment, with its associated long term side effects, ${ }^{10}$ is often unacceptable to first episode patients. ${ }^{18}$ Currently, decisions and guidelines on discontinuation must be made without directly relevant, randomised, placebo controlled data. In addition, no robust predictors exist to identify patients at low risk of relapse. ${ }^{23}$

\section{METHODS}

This double blind randomised controlled study aimed to investigate the effect of discontinuing maintenance treatment in a group of first episode patients who had completed at least one year of antipsychotic drug treatment with no relapses. Additionally, to optimise the clinical relevance of the study and the likelihood of successful discontinuation, we enrolled only patients clinically judged to be free of psychotic symptoms for at least eight weeks. Our primary hypothesis was that even for this group of patients selected for their good response, more patients randomised from existing treatment to placebo (discontinuation) than patients randomised to maintenance treatment (standardised to quetiapine) would relapse during one year of follow-up. We investigated readmission to hospital as a secondary outcome and also did an exploratory analysis of potential predictors of relapse.

\section{Study sites and participants}

Psychiatric care for first episode psychosis in Hong Kong is provided by specialised teams covering the entire population of approximately seven million. The Early Assessment Service for Young People with Psychosis (EASY) evaluates more than 600 new cases a year and provides comprehensive, guideline based case management for the first three years of illness. ${ }^{24}$ To enter the EASY programme, patients must have first episode psychosis, defined by continuously present psychotic symptoms since onset, no previous psychotic episodes, and no previous treatment or remission.

Inclusion criteria for the study included a diagnosis of schizophrenia or non-affective psychosis (schizophreniform disorder, schizoaffective disorder, brief psychotic disorder, or psychosis not otherwise specified) according to the Diagnostic and Statistical Manual for Mental Disorders, fourth edition (DSM-IV).$^{25}$ Experienced (equivalent to royal college or board certified) psychiatrists confirmed the diagnosis according to the Chinese version of the structured clinical interview for DSM-IV. ${ }^{26}$ In addition, patients had to be aged 18 to 65 , to have received antipsychotic drug treatment continuously for at least 12 months, and to have no history of relapse (defined as any increase in positive symptoms leading to adjustment of drug treatment or readmission to hospital). We ascertained this with information from patients, carers, case managers, clinicians, and clinical records. Patients had to be nonpsychotic at study entry, as defined by having below threshold scores on five key psychotic symptoms on the positive and negative syndrome scale (table 1$),{ }^{21}$ as well as free of positive symptoms of psychosis for at least eight weeks. Patients also had to score 2 (borderline or questionable mental illness) or below on the clinical global impressions scale. ${ }^{27}$

Exclusion criteria were drug induced psychosis and current treatment with clozapine or depot antipsychotics or with mood stabilising drugs (lithium, valproate, or carbamazepine). Other exclusion criteria included poor adherence to treatment (missing $>50 \%$ of drug, $>50 \%$ non-attendance at follow-up clinic visits, or a history of patient initiated discontinuation of treatment), and risk of suicide or violence. 
All participants gave written informed consent. All study participants were monitored by specialised clinical teams, as well as by the research team, and had access to a dedicated telephone hotline.

\section{Randomisation and blinding}

We randomised patients to maintenance with quetiapine $(400 \mathrm{mg} /$ day) or placebo of identical appearance for the next 12 months. AstraZeneca generated a randomisation sequence by computer, with a fixed block size of four without stratification. AstraZeneca prepared individually numbered sets of study drugs, packed them according to the randomisation sequence, and then shipped them to the study team in numbered but apparently identical sets. Study investigators assigned the study drug sets to participants consecutively according to the sequence of study entry. Investigators, patients, and all research staff were blind to the study drugs and the block size.

\section{Study design}

Patients had been treated with several different antipsychotic drugs for the 12 months before screening for entry to the study (table 2). We chose to randomise patients to a single antipsychotic drug or to placebo for prevention of relapse. This strategy avoids possible differences between subgroups of patients taking different maintenance drugs. Atypical antipsychotics are increasingly chosen for maintenance treatment in first episode psychosis. Among the three atypical antipsychotics available in Hong Kong at the time we started the study, we were able to obtain matching placebo tablets from the manufacturer only for quetiapine. We chose a dose of $400 \mathrm{mg} /$ day on the basis of the usual effective dose in patients with chronic illness; this dose has been shown to be efficacious for first episode patients. ${ }^{28}$

\begin{tabular}{|c|c|c|}
\hline Assessments & $\begin{array}{l}\text { Score indicating } \\
\text { remission }\end{array}$ & $\begin{array}{l}\text { Threshold score indicating } \\
\text { relapse }\end{array}$ \\
\hline \multicolumn{3}{|l|}{ Specific items $\left(\right.$ PANSS $\left.^{21}\right) \dagger:$} \\
\hline P1: Delusions & $\leq 2$ & 3 \\
\hline P2: Conceptual disorganisation & $\leq 3$ & 4 \\
\hline P3: Hallucinatory behaviour & $\leq 2$ & 3 \\
\hline P6: Suspiciousness & $\leq 4$ & 5 \\
\hline G9: Unusual thought content & $\leq 3$ & 4 \\
\hline \multicolumn{3}{|l|}{ Global scores $\left(\mathrm{CGI}^{27}\right) \ddagger$} \\
\hline Severity of illness & $\leq 2$ & 3 \\
\hline Improvement & NA & 5 \\
\hline \multicolumn{3}{|c|}{ 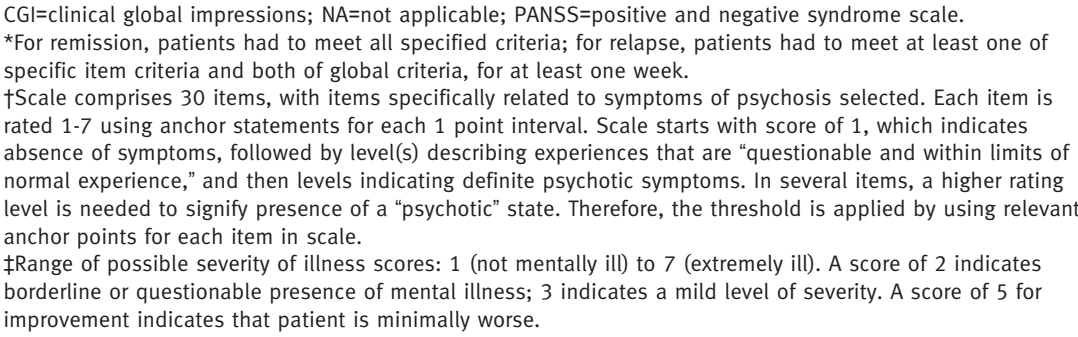 } \\
\hline
\end{tabular}

We achieved transition to the study drug by crosstapering over four to six weeks. We then assessed patients 12 times in the following year at approximately monthly intervals or until they had a relapse. We assessed adherence to treatment by clinical interviews with participants and informants, as well as by pill counting.

\section{Outcome measures}

As patients were non-psychotic at entry to the study, we defined relapse as the reappearance of definite psychotic symptoms (beyond ratings of "questionable" or "within limits of normal experience") according to predefined thresholds (table 1), together with a clinical global impressions score of 3 or above. This approach is consistent with earlier studies of relapse ${ }^{29}$; it represents a categorical shift in illness state. Our definitions are similar to the "exacerbation" described by Gitlin et al and are more liberal in defining relapse than the requirement for "substantial" or "unequivocal" deterioration or readmission to hospital used in other studies. ${ }^{10-12} 19$ The lower threshold for defining relapse allowed recurrence of psychotic symptoms to be recognised early and appropriate interventions to be started. We used alternative thresholds for defining relapse in secondary analyses (see below). Nine trained clinicians assessed the inter-rater reliability for each of the positive symptoms by using an independent sample of 20 patients (weighted $\kappa=0.72-0.92$ ). A secondary relapse related measure was readmission to hospital. To assess the functional implications of relapse, employment status (employed or otherwise) was reported at baseline and follow-up. Table 3 describes potential predictor variables for relapse and their measurements.

\section{Side effects and adverse events}

We assessed side effects monthly with the Udvalg for Kliniske Undersøgelser scale. ${ }^{36}$ We assessed movement disorders with the Barnes akathisia rating scale ${ }^{37}$ the abnormal involuntary movement scale, ${ }^{38}$ and the Simpson Angus scale. ${ }^{39}$ We measured body weight and body mass index at baseline and at six and 12 months. We defined adverse events as adverse occurrences that may have been related to the drug and serious adverse events as death, hospital admission, or persistent incapacity.

\section{Statistical analysis}

We estimated statistical power according to Collett (1994), ${ }^{40}$ by first determining the required number of outcome events (relapses). Because some relapses during the transition from drug at entry to study drug could be related to the transition itself, we planned to ensure that the comparison of post-transition relapses would have sufficient power. No controlled study data for this group had been reported in the literature at the time we were planning the study; naturalistic studies suggested a range of relapse rates from $30 \%$ to $55 \%$. We aimed to be able to detect an effect that would 
Table 2 | Baseline drug treatment. Values are numbers (percentages) unless stated otherwise

\begin{tabular}{|c|c|c|c|}
\hline Characteristics & Quetiapine group $(n=89)$ & Placebo group $(n=89)$ & All patients $(n=178)$ \\
\hline $\begin{array}{l}\text { Median (interquartile range) duration of } \\
\text { antipsychotic treatment before study (days) }\end{array}$ & 647 (472 to 828$)$ & 589 (471 to 779) & $618(472$ to 784$)$ \\
\hline \multicolumn{4}{|l|}{ Antipsychotic drugs: } \\
\hline Typical antipsychotics & $41(46)$ & $45(51)$ & $86(48)$ \\
\hline Atypical antipsychotics & $48(54)$ & $44(49)$ & $92(52)$ \\
\hline Risperidone & $14(16)$ & $22(25)$ & $36(20)$ \\
\hline Olanzapine & $13(15)$ & $14(16)$ & $27(15)$ \\
\hline Quetiapine & $14(16)$ & $3(3)$ & $17(10)$ \\
\hline Amisulpride & $7(8)$ & $4(4)$ & $11(6)$ \\
\hline Ziprasidone & 0 & $1(1)$ & $1(1)$ \\
\hline \multicolumn{4}{|l|}{ Mean (SD) doses (mg/day): } \\
\hline Typical antipsychotics* & $153.0(193.9)$ & $125.8(92.3)$ & $138.8(149.3)$ \\
\hline \multicolumn{4}{|l|}{ Atypical antipsychotics } \\
\hline Risperidone (n=36) & $2.1(1.3)$ & $2.0(1.1)$ & $2.1(1.2)$ \\
\hline Olanzapine $(\mathrm{n}=27)$ & $5.8(4.8)$ & $7.1(3.4)$ & $6.5(4.1)$ \\
\hline Quetiapine (n=17) & $271.4(184.7)$ & $140.0(138.9)$ & $248.2(181.1)$ \\
\hline Amisulpride (n=11) & $264.3(102.9)$ & $200.0(81.7)$ & $240.9(97.0)$ \\
\hline Ziprasidone $(n=1)$ & NA & 40 & 40 \\
\hline \multicolumn{4}{|l|}{ Concurrent drugs: } \\
\hline Antidepressants & $20(22)$ & $16(18)$ & $36(20)$ \\
\hline Anticholinergics & $11(12)$ & $10(11)$ & $21(12)$ \\
\hline Benzodiazepines & $3(3)$ & $4(4)$ & $7(4)$ \\
\hline
\end{tabular}

result in a post-transition relapse rate of $45 \%$ in the discontinuation group and $25 \%$ in the maintenance group (a hazard ratio of 0.48 ). To detect this effect with a type I error of 0.05 (two tailed), and a power of 0.8 , we needed a total of at least 58 post-transition relapses. ${ }^{40}$ We therefore planned to recruit patients until at least 58 post-transition relapses took place. We stopped recruitment when 60 post-transition relapses had occurred in the study, after 178 patients had been randomised. Subsequent to our data collection, a randomised open label study of discontinuation versus maintenance treatment reported relapse rates of $43 \%$ and $21 \%$, giving a hazard ratio of $0.42 .{ }^{20}$ Our study would have a power of 0.9 in detecting a similar effect.

We used SPSS software (version 16.0) for the statistical analysis. All tests were two tailed with a significance level of $\mathrm{P}<0.05$. We included all completers (either reaching the end of the study or relapsing) and all discontinued participants. The primary outcome measure was reappearance of definite psychotic symptoms. The positive and negative syndrome scale scores of two patients were missing at the point of relapse (as they were admitted to hospital before the research team was notified), but in both cases clinical information indicated that they were experiencing considerable hallucinations and delusions. We assigned both cases as relapses and treated them as such in the survival analysis.

We calculated the relapse-free survival time from randomisation to the last visit or relapse. Patients who discontinued from the study (mostly because of adverse events) continued to be managed by their usual clinical teams. Their team physicians decided whether to continue further maintenance treatment while remaining blinded to the patients' study treatments (active or placebo). As this trial specific scenario would not occur in routine clinical practice, we censored data from patients who discontinued at the time of discontinuation in the primary survival analysis. This approach is acceptable for an explanatory efficacy study to provide the most meaningful estimate of the effect of maintenance treatment with the study drug. ${ }^{41}$ We used the Kaplan-Meier method to construct survival curves and the log-rank test to compare them.

We planned a post-transition subgroup analysis excluding early relapses (occurring within 60 days of randomisation, and thus possibly related to switching medication). Two post hoc sensitivity analyses assessed the impact of more stringent criteria for relapse: two or more psychotic symptoms above threshold, and any psychotic symptom with a score of 5 (moderately severe) or above. We censored patients with less severe psychotic symptoms. A secondary post hoc analysis evaluated the rates of hospital admission for all causes in the two treatment groups (Fisher's exact test for small number of counts). Two further post hoc sensitivity analyses evaluated the effects of including estimates for post-discontinuation data. In the first analysis, we estimated the worst case scenario by treating all discontinuations as relapses. ${ }^{42}$ In the second analysis, we made a clinically pragmatic estimate by using data from case notes about relapses for the period after discontinuation from the study. In addition, we categorised patients into four groups on 
the basis of changes in their occupational functioning: from employed to unemployed, from unemployed to employed, employed with no change, and unemployed with no change. We used $\chi^{2}$ tests to compare the proportion of participants with these changes between the two treatment groups.

We compared discontinuations due to all causes and those due to adverse events and serious adverse events by using relative risk and $\chi^{2}$ tests. We compared side effects at baseline and at follow-up by using $\chi^{2}$ tests or Fisher's exact test if the number of side effects in a group was less than five. We compared body weight and body mass index between the maintenance and placebo groups by using $t$ tests both at baseline and at the end of the study. We used $\chi^{2}$ tests to compare the proportions of participants with body mass index of 23 or above and with weight gain of $7 \%$ or more between the two groups. Lastly, we further compared changes in weight or body mass index between the two groups by linear regression analysis, with the change as the dependent variable, the treatment group denoted by an indicator, and adjustment by weight or body mass index at baseline by group and previous drug type (typical or atypical). We validated the fitted models by diagnostic residual plots.

We used univariate Cox proportional hazards models to analyse the potential predictors of relapse. We included variables with $\mathrm{P} \leq 0.1$ in a multivariate $\mathrm{Cox}$ regression analysis with forward selection. We assessed the proportional hazard assumption by the method described in Lin et al, ${ }^{43}$ using SAS 9.1.3. We checked associations between the predictors by using
Pearson correlation (continuous variables), $\chi^{2}$ test (categorical variables), or Wilcoxon rank sum test (continuous and categorical variables). We re-fitted the selected Cox model to all patients with non-missing predictors to obtain more precise estimates. We calculated relative risk as the ratio between the outcome (relapse) rate in the population exposed to the risk factor (placebo) divided by the outcome rate in the population not exposed to the risk factor (maintenance quetiapine).

\section{RESULTS}

Study population

Between September 2003 and July 2006, we assessed consecutive patients already enrolled in the early psychosis programme for eligibility to participate in the relapse prevention study. We screened 1606 patients for eligibility (fig 1). Schizophrenia was the most common diagnosis in enrolled patients (table 4). Before randomisation, patients had received antipsychotic drugs for a median of 21 months (618 (interquartile range 472-784) days) (table 2). The total symptom severity scores were low, and the median global severity of illness rating was 1 , or "not mentally ill." The level of functioning as assessed by the rating scales and proportion employed was moderately high. Baseline variables in the group randomised to quetiapine did not differ from those randomised to placebo (all $\mathrm{P}>0.05)$. Including patients who completed the study, relapsed, or were withdrawn, the median duration of follow-up was 145 (41-351) days for the quetiapine group and 106 (57-243) days for the placebo group.

Table $3 \mid$ Predictors of relapse in univariate and multivariate Cox proportional hazards regression analysis $(\mathrm{n}=178)$

\begin{tabular}{|c|c|c|c|c|}
\hline \multirow[b]{2}{*}{ Predictors } & \multicolumn{2}{|c|}{ Univariate analysis* } & \multicolumn{2}{|c|}{ Multivariate analysis $(n=160)$} \\
\hline & Hazard ratio $(95 \% \mathrm{Cl})$ & $P$ value & Hazard ratio $(95 \% \mathrm{Cl})$ & $P$ value \\
\hline Treatment (quetiapine or placebo) & $0.41(0.26$ to 0.65$)$ & $<0.001$ & $0.37(0.23$ to 0.60$)$ & $<0.001$ \\
\hline Schizophrenia diagnosis $\dagger$ & 1.83 (1.18 to 2.85$)$ & 0.01 & $1.72(1.07$ to 2.76$)$ & 0.03 \\
\hline $\mathrm{PSST}^{32} 1$ unit $(\mathrm{n}=160)$ & 1.87 (1.16 to 3.02$)$ & 0.01 & $1.86(1.15$ to 3.00$)$ & 0.01 \\
\hline SOFAS, ${ }^{30} \ddagger 1$ unit & 0.98 (0.96 to 0.99$)$ & 0.01 & 0.98 (0.96 to 1.00$)$ & 0.05 \\
\hline Sex & $1.11(0.72$ to 1.71$)$ & 0.64 & NA & \\
\hline Age of onset, year & $1.00(0.97$ to 1.03$)$ & 0.84 & NA & \\
\hline Age, year & $1.00(0.97$ to 1.03$)$ & 0.99 & NA & \\
\hline Education, year & 0.95 (0.89 to 1.02$)$ & 0.18 & NA & \\
\hline SANS, ${ }^{33} 1$ unit & 1.01 (1.00 to 1.03$)$ & 0.05 & NA & \\
\hline SAPS, ${ }^{34} 1$ unit & $0.87(0.67$ to 1.12$)$ & 0.28 & NA & \\
\hline SUMD, ${ }^{35} 1$ unit $(n=159)$ & $1.10(0.98$ to 1.24$)$ & 0.09 & NA & \\
\hline PAS, ${ }^{31} \S 1$ unit $(n=157)$ & $1.24(0.98$ to 1.58$)$ & 0.08 & NA & \\
\hline Concomitant treatment & $1.53(0.96$ to 2.43$)$ & 0.08 & NA & \\
\hline Duration of untreated psychosis, day & 1.00 (1.00 to 1.00$)$ & 0.51 & NA & \\
\hline Duration of previous antipsychotic treatment, day & $1.00(1.00$ to 1.00$)$ & 0.25 & NA & \\
\hline Type of previous antipsychotic treatment (atypical or typical) & $0.84(0.55$ to 1.31$)$ & 0.45 & NA & \\
\hline
\end{tabular}

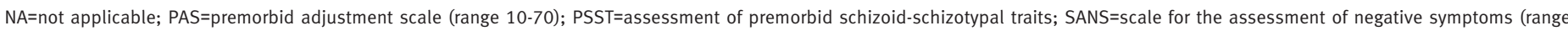
$0-120)$; SAPS=scale for the assessment of positive symptoms (range 0-175); SOFAS=social and occupational functioning assessment scale (range $1-100$ ); SUMD=scale to assess unawareness of mental disorder (range 3-9).

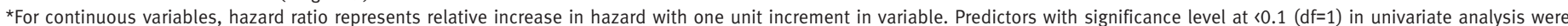
included in multivariate analysis.

†Categorised into schizophrenia and non-schizophrenia (including schizophreniform disorder, schizoaffective disorder, brief psychotic disorder, and psychosis not otherwise specified). Schizophrenia diagnosis was correlated with SANS ( $z=-4.122$; $P<0.001)$.

†SOFAS was correlated with SANS ( $r=-0.59 ; \mathrm{P}<0.001)$ and schizophrenia diagnosis $(z=-2.45 ; P=0.01)$.

§PAS was correlated with PSST ( $r=0.54 ; \mathrm{P}<0.001)$, SANS $(r=0.18 ; \mathrm{P}=0.02)$ and SOFAS $(r=-0.17 ; \mathrm{P}=0.03)$ 


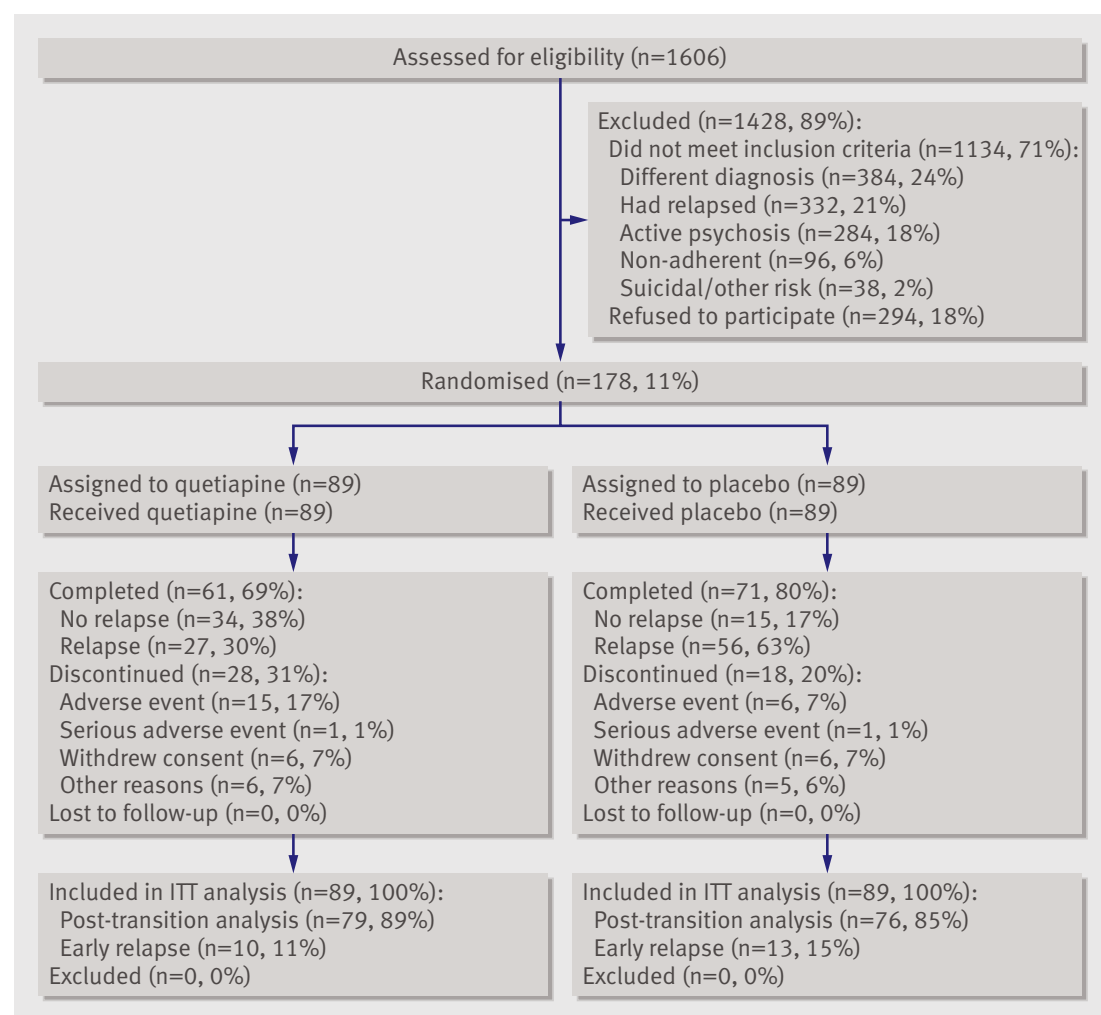

Fig 1| Enrolment and outcomes. ITT=intention to treat

\section{Primary outcome: relapse}

Twenty-seven of 89 patients relapsed in the quetiapine group, and 56 of 89 patients relapsed in the placebo group. The Kaplan-Meier estimate of the proportion relapsed at 12 months was $41 \%$ (95\% confidence interval $29 \%$ to $53 \%)$ in the quetiapine group and $79 \%(68 \%$ to $90 \%)$ in the placebo group $\left(\chi^{2}=15.65, \mathrm{df}=1 ; \mathrm{P}<0.001\right.$ by log-rank) (fig 2). In the post-transition subgroup analysis excluding the 23 patients who relapsed within 60 days, fewer patients in the quetiapine group relapsed (22\% (17/79); 12 month Kaplan-Meier estimate $32 \%, 19 \%$ to $45 \%)$ than in the placebo group $(57 \%(43 / 76) ; 75 \%, 62 \%$ to $89 \%) \quad\left(\chi^{2}=19.17, \mathrm{df}=1\right.$; $\mathrm{P}<0.001$ by $\log$-rank).

In relapses, specific symptoms that scored at or above the threshold included delusions $(72 \% ; 58 / 81)$, hallucinations $(54 \% ; 44 / 81)$, suspiciousness $(38 \%$; $31 /$ $81)$, conceptual disorganisation $(30 \% ; 24 / 81)$, and unusual thought content $(23 \% ; 19 / 81) ; 68 \%(55 / 81)$ of patients had two or more types of psychotic symptom. The most common co-occurring psychotic symptoms were delusions and hallucinations $(37 \% ; 30 / 81)$ and delusions and suspiciousness $(31 \% ; 25 / 81)$. The magnitude of the highest score of the five key symptoms defining relapse did not differ between relapsing patients in the two treatment groups (mean (SD) 4.30 (0.91) in quetiapine group ( $\mathrm{n}=27)$ v $4.50(0.82)$ in placebo group $(\mathrm{n}=54) ; t=-1.02, \mathrm{df}=79 ; \mathrm{P}=0.31)$. Nor did we find a difference in the magnitude of the change in score from baseline for these symptoms (mean (SD) 3.22 (1.01) in quetiapine group $v 3.46(0.82)$ in placebo group; $t=-1.15, \mathrm{df}=79 ; \mathrm{P}=0.25)$. We found no significant correlation between severity of symptoms at relapse and the time to receiving treatment $(\mathrm{r}=$ $-0.37, \mathrm{P}=0.06$ for quetiapine group; $\mathrm{r}=0.07, \mathrm{P}=0.61$ for placebo group).

We did two sensitivity analyses defining relapse with more stringent criteria: two or more psychotic symptoms above the threshold, and requiring a score of 5 (moderately severe) to define relapse. We censored patients with less severe psychotic symptoms. When we used the first criterion, the quetiapine group showed a significantly lower relapse rate $(30 \%, 95 \%$ confidence interval $18 \%$ to $42 \%$ ) than did the placebo group $(68 \%, 56 \%$ to $80 \%)\left(\chi^{2}=14.67, \mathrm{df}=1 ; \mathrm{P}<0.001\right.$ by log-rank). When we used the second criterion, the quetiapine group also showed a significantly lower relapse rate $(27 \%, 15 \%$ to $39 \%)$ than did the placebo group $(63 \%, 46 \%$ to $79 \%)\left(\chi^{2}=11.81, \mathrm{df}=1 ; \mathrm{P}=0.001\right.$ by $\log$ rank).

In the post hoc sensitivity analysis of the worst case scenario, we treated all discontinuations/dropouts as having relapsed at point of discontinuation. The Kaplan-Meier estimate of the proportion of relapse at 12 months was $62 \%(51 \%$ to $72 \%)$ in the quetiapine group and $85 \%(76 \%$ to $93 \%)$ in the placebo group $\left(\chi^{2}=5.59, \mathrm{df}=1 ; \mathrm{P}=0.018\right.$ by log-rank $)$. We did another post hoc sensitivity analysis with information from case notes about relapses for the post-discontinuation period. This provides a pragmatic estimate by including all clinically significant relapses in the post-discontinuation period. The Kaplan-Meier estimate of the proportion of relapse at 12 months was $41 \%$ (30\% to $51 \%$ ) in the quetiapine group and $69 \%(59 \%$ to $79 \%)$ in the placebo group $\left(\chi^{2}=14.25, \mathrm{df}=1 ; \mathrm{P}<0.001\right.$ by log-rank $)$.

\section{Secondary outcome measures}

Readmission to hospital was more common in the placebo group (16\% (14/89); 12 relapse, 2 serious adverse

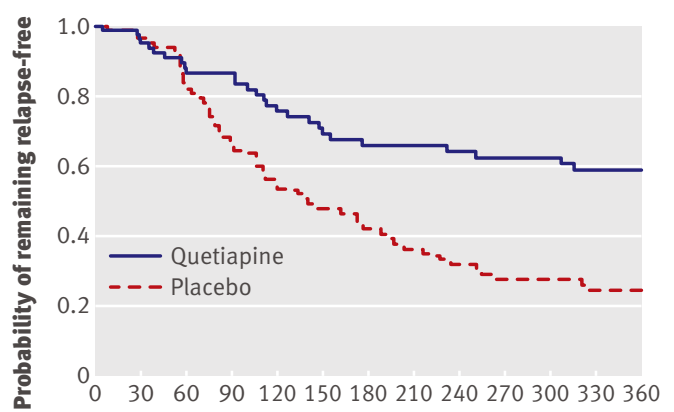

No at risk

Quetiapine $89 \begin{array}{lllllllllll}71 & 58 & 56 & 49 & 42 & 39 & 38 & 37 & 36 & 36 & 31\end{array}$ $\begin{array}{llllllllllllll}\text { Placebo } & 89 & 76 & 62 & 49 & 39 & 33 & 29 & 25 & 22 & 19 & 18 & 16\end{array}$

\section{No with relapse}

$\begin{array}{lllllllllllll}\text { Quetiapine } & 0 & 4 & 10 & 10 & 17 & 21 & 23 & 23 & 24 & 25 & 25 & 27\end{array}$ $\begin{array}{lllllllllllll}\text { Placebo } & 0 & 3 & 13 & 25 & 35 & 39 & 43 & 47 & 50 & 53 & 53 & 55\end{array}$

Fig 2 | Kaplan-Meier analysis in remitted first episode psychosis patients with and without antipsychotic maintenance treatment. Median duration of follow-up was 145 (interquartile range 41-351) days for quetiapine group and 106 (57-243) days for placebo group 
Table $4 \mid$ Baseline characteristics of patients. Values are numbers (percentages) unless stated otherwise

\begin{tabular}{|c|c|c|c|}
\hline Characteristics & $\begin{array}{l}\text { Quetiapine group } \\
\qquad(n=89)\end{array}$ & $\begin{array}{l}\text { Placebo group } \\
\quad(n=89)\end{array}$ & All patients $(n=178)$ \\
\hline Mean (SD) age (years) & $23.5(5.2)$ & $24.9(7.3)$ & $24.2(6.4)$ \\
\hline Mean (SD) age at onset (years) & $21.4(5.3)$ & $22.4(7.5)$ & $21.9(6.5)$ \\
\hline Male sex & $39(44)$ & $41(46)$ & $80(45)$ \\
\hline Mean (SD) education (years) & $12.0(2.6)$ & $11.7(3.1)$ & $11.8(2.8)$ \\
\hline Employed & $62(70)$ & $65(73)$ & $127(71)$ \\
\hline \multicolumn{4}{|l|}{ Type of disorder: } \\
\hline Schizophrenia & $46(52)$ & $45(51)$ & $91(51)$ \\
\hline Schizophreniform disorder & $15(17)$ & $12(13)$ & $27(15)$ \\
\hline Schizoaffective disorder & $3(3)$ & $6(7)$ & $9(5)$ \\
\hline Brief psychotic disorder & $13(15)$ & $8(9)$ & $21(12)$ \\
\hline Psychosis not otherwise specified & $12(13)$ & $18(20)$ & $30(17)$ \\
\hline \multicolumn{4}{|l|}{ Comorbid diagnoses: } \\
\hline Affective disorder & $5(6)$ & $2(2)$ & $7(4)$ \\
\hline Previous substance abuse & $2(2)$ & $4(4)$ & $6(3)$ \\
\hline Personality disorder & 0 & $1(1)$ & $1(1)$ \\
\hline Others & $1(1)$ & 0 & $1(1)$ \\
\hline \multicolumn{4}{|l|}{ Mean (SD) PANSS ${ }^{21}$ sum score $(n=177)$ : } \\
\hline Total & $36.1(4.6)$ & $37.1(6.4)$ & $36.6(5.6)$ \\
\hline Positive & $7.2(0.6)$ & $7.3(0.7)$ & $7.2(0.6)$ \\
\hline Negative & $8.7(2.9)$ & $9.1(3.6)$ & $8.9(3.3)$ \\
\hline Mean (SD) $\mathrm{CGI}^{27}$ severity score $(\mathrm{n}=176)$ & $1.1(0.3)$ & $1.2(0.4)$ & $1.1(0.3)$ \\
\hline Mean (SD) SOFAS ${ }^{30}$ score* $^{\star}$ & $76.4(10.0)$ & $74.6(11.7)$ & $75.5(10.9)$ \\
\hline Mean (SD) PAS $31+(n=157)$ & $2.7(0.9)$ & $2.8(0.8)$ & $2.8(0.9)$ \\
\hline Mean (SD) PSST $^{32} \ddagger(n=160)$ & $1.5(0.5)$ & $1.5(0.4)$ & $1.5(0.5)$ \\
\hline
\end{tabular}

$\mathrm{CGI}=$ clinical global impressions; PANSS=positive and negative syndrome scale; $\mathrm{PAS}=$ premorbid adjustment scale; PSST=assessment of premorbid schizoid and schizotypal traits; SOFAS=social and occupational functioning assessment scale.

*Range of scores from 1 to 100 , with lower scores representing impaired functioning.

†Measures functional level before onset of illness, with range of scores from 10 to 70 and lower scores indicating better functioning.

¥Measures premorbid personality in patients before first episode psychosis on basis of interview of an informant, with range of scores from 8 to 32 and lower scores indicating fewer schizoid-schizotypal traits.

events) than in the quetiapine group $(6 \%(5 / 89) ; 4$ relapse, 1 serious adverse event) (Fisher's exact test (two tailed) $\mathrm{P}<0.05)$. The groups did not differ in baseline occupational status-open employment: quetiapine group 70\% (62/89); placebo group 73\% (65/89). Some participants lost open employment status in the study period - quetiapine group 27\% (17/62); placebo group 32\% (21/65). Changes in occupational status (from employed to unemployed, from unemployed to employed, employed with no change, or unemployed with no change) during the study did not differ statistically between the two treatment groups $\left(\chi^{2}=1.52, \mathrm{df}=3 ; \mathrm{P}=0.68\right)$.

\section{Predictors of relapse}

We studied predetermined potential predictors of relapse initially with a univariate Cox regression model. We then entered variables with $\mathrm{P}$ values of 0.1 or below into a multivariate Cox regression. We found the assumption of proportionality to be valid. Significant independent predictors of relapse were maintenance treatment, pre-morbid schizoid and schizotypal traits, functioning, and diagnosis of schizophrenia (table 3).
Side effects, adverse events, and discontinuations Patients taking quetiapine reported more side effects (sleepiness or sedation, reduced salivation, and constipation) than did those taking placebo (table 5). Only $11 \%(20 / 178 ; 9$ in quetiapine group and 11 in placebo group) of patients had a Simpson Angus scale score above zero at baseline. Those previously treated with typical antipsychotics had higher mean scores $(\mathrm{P}=0.02)$. Most $(145 / 168 ; 76$ in quetiapine group and 69 in placebo group) had no change in these scores during the study. Five had a higher score (four in placebo group, one in quetiapine group), and 18 had lower scores (nine in each group). We found no difference in the change in scores between the two groups. Only two participants had positive abnormal involuntary movement scale scores at baseline (one in quetiapine group, one in placebo group); both scores decreased during the study. Two other patients in the placebo group (one previously treated with a typical antipsychotic and one with an atypical antipsychotic) had an increase in this score during the study. Four patients had positive scores for akathisia at baseline (all in placebo group), and six had positive scores at follow-up (four in quetiapine group, two in placebo group). Six patients had an increased score (four in quetiapine group, two in placebo group), and four had a reduced score at follow-up (all in placebo group).

Table 6 shows changes in body weight and body mass index; it also shows some summary statistics by subsets. We found an overall trend for weight reduction, which was greater in the placebo group (mean (SD) $-3.08(8.22) \mathrm{kg}$ ) than in the quetiapine group $(-0.75(4.65) \mathrm{kg})(\mathrm{P}=0.02)$. Baseline weight had a significant effect on the change in weight for the placebo group; heavier patients at baseline had more weight reduction (unstandardised regression coefficient $-0.19,95 \%$ confidence interval -0.28 to -0.10 ; $\mathrm{P}<0.001)$. The baseline effect was not significant for the quetiapine group (regression coefficient -0.08 , -0.17 to $0.2 ; \mathrm{P}=0.11)$. Previous drug type had a minor effect on weight change (typical antipsychotic 1.72 $(-0.15$ to 3.59$) \mathrm{kg}$ more than atypical antipsychotic; $\mathrm{P}=0.07)$. Weight change over time did not differ between the groups after adjustment for weight at baseline (placebo group 5.39 (-3.22 to 14.0$) \mathrm{kg}$ more than quetiapine group; $\mathrm{P}=0.22$ ). The body mass index at baseline had a significant effect on the change in body mass index for the placebo group (regression coefficient $-0.25,-0.35$ to $-0.15 ; \mathrm{P}<0.001$ ) but not for the quetiapine group (regression coefficient $-0.06,-0.16$ to $0.40 ; \mathrm{P}=0.26$ ). Previous drug type had no significant effect on body mass index (typical antipsychotic 0.57 $(-0.12$ to 1.26$) \mathrm{kg}$ more than atypical; $\mathrm{P}=0.11)$, but the mean change in body mass index differed somewhat between the groups (placebo group 3.74 (0.17 to 7.31) $\mathrm{kg}$ more than quetiapine group; $\mathrm{P}=0.04)$ after adjustment for body mass index at baseline. No diabetes or ketoacidosis emerged during the study period.

Figure 1 gives reasons for discontinuation. Adverse events that occurred in more than one case were fatigue, sedation, or tiredness $(n=9 ; 7$ in quetiapine group, 
2 in placebo group), dizziness $(\mathrm{n}=7$; all in quetiapine group), nausea ( $\mathrm{n}=2$; both in quetiapine group), limb weakness ( $\mathrm{n}=2$; both in quetiapine group), and insomnia ( $\mathrm{n}=2 ; 1$ in quetiapine group, 1 in placebo group). Three serious adverse events occurred. One patient taking quetiapine was admitted to hospital after impulsive self-laceration following an argument with a partner, and one assigned to placebo was admitted after a non-fatal overdose of hypnotics during an adjustment reaction to financial difficulties. Both were discontinued. A third patient in the placebo group had transient repetitive imagery for three days and was briefly admitted for observation, but continued. The rate of discontinuation for both adverse events and serious adverse events was higher for the quetiapine group $(18 \% ; 16 / 89)$ than the placebo group $(8 \% ; 7 / 89)$ (relative risk $2.29,95 \%$ confidence interval 0.99 to 5.28 ; $\left.\chi^{2}=3.20, \mathrm{df}=1 ; \mathrm{P}=0.07\right)$.

Other reasons for discontinuation included need for a mood stabiliser ( $\mathrm{n}=3$; 2 in quetiapine group, 1 in placebo group), inability to tolerate the transition protocol ( $\mathrm{n}=7 ; 4$ in quetiapine group, 3 in placebo group), and unwillingness to attend the study assessments $(n=1$; in placebo group). Discontinuation for all causes did not differ significantly between the quetiapine group $(31 \%$; $28 / 89)$ and the placebo group $(20 \% ; 18 / 89)$ (relative risk $1.32,0.98$ to $1.77 ; \chi^{2}=2.38, \mathrm{df}=1 ; \mathrm{P}=0.12$ ).

\section{DISCUSSION}

In patients with first episode psychosis, antipsychotic treatment is often discontinued after one year of good response. We investigated patients selected from a representative, population based sample. This group of patients represents those with the best chance of successful discontinuation of treatment. We found a much higher rate of relapse in patients randomised to placebo $(79 \%)$ than in those receiving active maintenance treatment $(41 \%)$. A subgroup analysis excluding patients with early relapse (possibly related to transition to the study drug), sensitivity analyses, and comparison of rates of admission to hospital were all consistent with the primary outcome analysis.

\section{Comparison with other studies}

The rate of relapse in the placebo group in our study is higher than in some studies, ${ }^{1012}$ but it is lower than in others. ${ }^{11}{ }^{19}$ The relapse rate in the maintenance group is comparable to that in previous studies. ${ }^{10}{ }^{12}$ Our definition of relapse signified the re-emergence of psychotic symptoms in patients who were not psychotic at baseline, consistent with the approach of Vaughn et al. ${ }^{29}$ This definition of relapse is relevant to real life decisions about treatment, as most clinicians would recommend resumption of antipsychotic treatment in this situation. As in other studies with intensive follow-up and monitoring, ${ }^{1920}$ relapses were detected relatively

\begin{tabular}{|c|c|c|c|c|c|c|}
\hline \multirow[b]{2}{*}{ Side effects } & \multicolumn{3}{|c|}{ Baseline } & \multicolumn{3}{|c|}{ Study period } \\
\hline & $\begin{array}{l}\text { Quetiapine } \\
\text { group }(n=89)\end{array}$ & $\begin{array}{l}\text { Placebo group } \\
\quad(n=89)\end{array}$ & $P$ value* & $\begin{array}{l}\text { Quetiapine } \\
\text { group }(n=89)\end{array}$ & $\begin{array}{l}\text { Placebo group } \\
\quad(n=89)\end{array}$ & P value* $^{*}$ \\
\hline \multicolumn{7}{|l|}{$\mathrm{UKU}^{36}+:$} \\
\hline Concentration difficulties & $9(10)$ & $23(26)$ & 0.01 & $37(42)$ & $47(53)$ & 0.18 \\
\hline $\begin{array}{l}\text { Asthenia, lassitude, or } \\
\text { increased fatigability }\end{array}$ & $19(21)$ & $24(27)$ & 0.48 & $52(58)$ & $46(52)$ & 0.45 \\
\hline Sleepiness or sedation & $23(26)$ & $19(21)$ & 0.60 & $62(70)$ & $44(49)$ & 0.01 \\
\hline Failing memory & $7(8)$ & $14(16)$ & 0.16 & $33(37)$ & $30(34)$ & 0.75 \\
\hline Depression & $1(1)$ & $1(1)$ & $>0.99$ & $16(18)$ & $12(13)$ & 0.54 \\
\hline Tension or inner unrest & $2(2)$ & $1(1)$ & $>0.99$ & $19(21)$ & $14(16)$ & 0.44 \\
\hline Increased duration of sleep & $10(11)$ & $13(15)$ & 0.66 & $45(51)$ & $35(39)$ & 0.18 \\
\hline Reduced duration of sleep & $1(1)$ & $1(1)$ & $>0.99$ & $22(25)$ & $30(34)$ & 0.25 \\
\hline Tremor & $4(4)$ & $4(4)$ & $>0.99$ & $16(18)$ & $13(15)$ & 0.69 \\
\hline Reduced salivation & $1(1)$ & 0 & $>0.99$ & $13(15)$ & $4(4)$ & 0.04 \\
\hline Constipation & $2(2)$ & 0 & 0.50 & $13(15)$ & $4(4)$ & 0.04 \\
\hline Weight gain & $22(25)$ & $19(21)$ & 0.72 & $35(39)$ & $30(34)$ & 0.54 \\
\hline Weight loss & 0 & $1(1)$ & $>0.99$ & $9(10)$ & $23(26)$ & 0.01 \\
\hline Amenorrhoea & $6 / 50(12)$ & $5 / 48(10)$ & 0.77 & $12 / 50(24)$ & $11 / 48(23)$ & $>0.99$ \\
\hline Headache & $4(4)$ & $2(2)$ & 0.68 & $19(21)$ & $16(18)$ & 0.71 \\
\hline Akathisia $\left(\right.$ BARS $\left.^{37}\right) \ddagger$ & 0 & 0 & $>0.99$ & $1(1)$ & 0 & $>0.99$ \\
\hline Dyskinesia $\left(\mathrm{AIMS}^{38}\right) \S$ & 0 & 0 & $>0.99$ & 0 & $1(1)$ & 0.48 \\
\hline Parkinsonism $\left(\mathrm{SAS}^{39}\right)$ ף & 0 & 0 & $>0.99$ & 0 & $1(1)$ & $>0.99$ \\
\hline
\end{tabular}

AIMS=abnormal involuntary movement scale; BARS=Barnes akathisia rating scale; SAS=Simpson Angus scale; UKU=Udvalg for Kliniske Undersøgelser scale.

*Based on $x^{2}$ comparisons between quetiapine and placebo groups, or Fisher's exact test when number of side effects in group is $<5$.

†Side effects scored as 1 (mild) or higher at baseline or at any time during the study period; extrapyramidal side effects were as defined in CATIE study, ${ }^{44}$ with thresholds adjusted for first episode patients.

‡BARS global clinical assessment score $\geq 2$, indicating akathisia of mild or greater severity.

$\S A I M S$ global severity score $\geq 2$, indicating abnormal involuntary movements of mild or greater severity.

ISAS mean score $\geq 1$, indicating extrapyramidal signs of moderate severity. 
Table 6 |Weight and body mass index (BMI) at baseline and changes during study period*

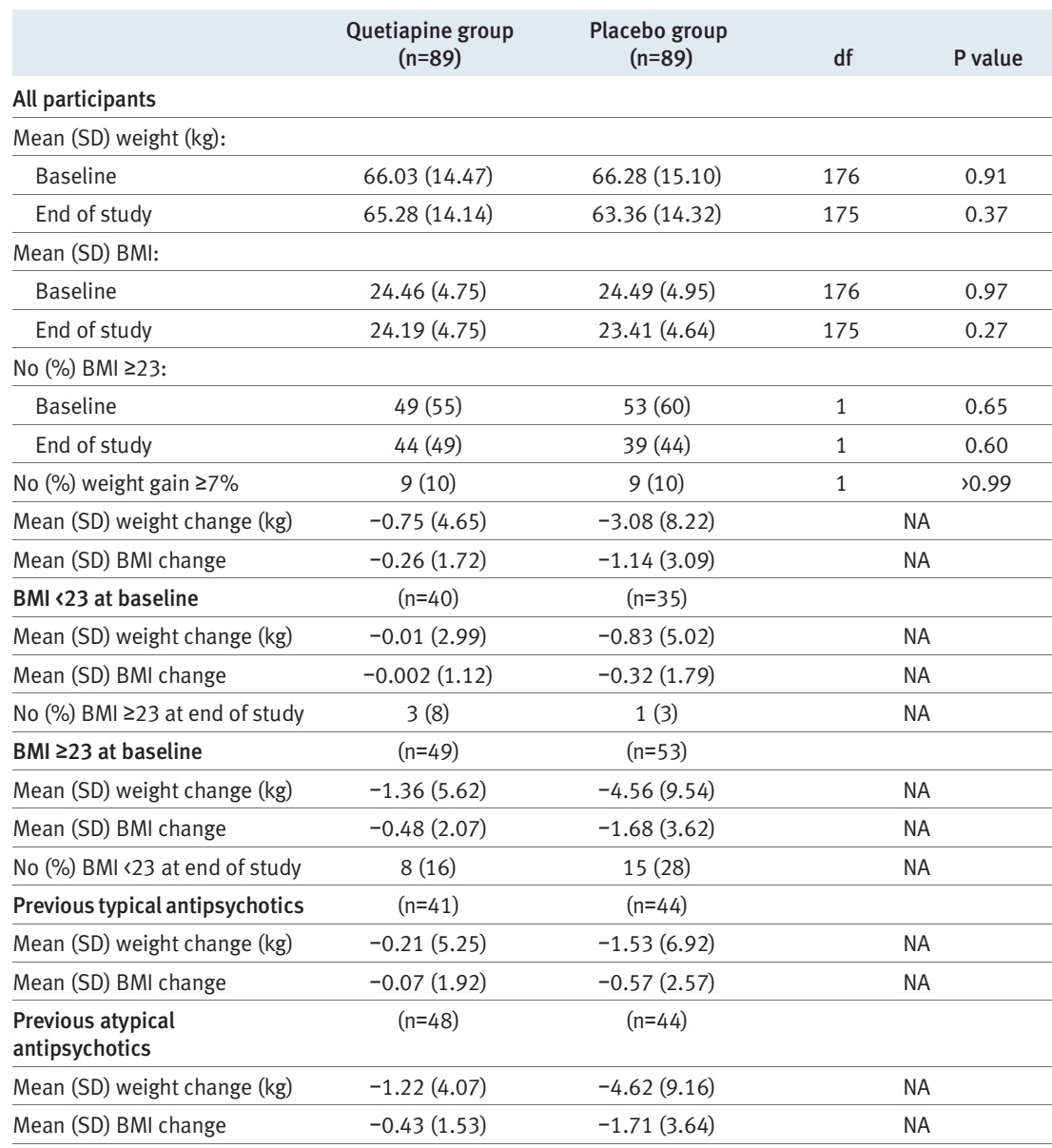

$\mathrm{NA}=$ not applicable.

early, in most cases before pronounced deterioration and need for hospital admission. The level of severity of symptoms that we used to define relapse is described in some other studies as an "exacerbation." ${ }^{19}$ However, the relapses into psychosis in our study were not trivial and in most cases involved two or more types of psychotic symptoms. Predictors of relapse included premorbid schizoid and schizotypal traits and low psychosocial functioning, as in earlier studies. ${ }^{1011}$ Importantly, we found that relapse was related neither to the type (atypical or typical) nor to the duration of previous antipsychotic treatment.

\section{WHAT IS ALREADY KNOWN ON THIS TOPIC}

After first episode psychosis, the decision on whether to discontinue antipsychotic drugs after a period of maintenance treatment is a common clinical problem

Little empirical evidence exists to guide physicians in this decision

\section{WHAT THIS STUDY ADDS}

Among first episode patients who have had a full remission and taken antipsychotic drugs for at least a year, the relapse rate after discontinuation was still high compared with those who continued treatment

These findings are decisive in supporting maintenance treatment, even after one year of maintenance and in patients with no positive symptoms of psychosis

\section{Limitations of study}

The study has several limitations. The choice of a design with a standardised drug treatment and a placebo control required switching of drugs. This design, however, has the advantages of randomisation and a double blind placebo control. Without a double blind control, the effects of patients' and clinicians' expectations cannot be assessed and the magnitude of the benefits of maintenance cannot be quantified. We evaluated the effects of switching by a subgroup analysis that excluded patients who relapsed within two months of the switch period. The 12 month KaplanMeier estimate of a $41 \%$ relapse rate in the primary analysis included patients who might have relapsed as a result of switching in itself. The post-transition subgroup analysis, which excluded patients who relapsed early, resulted in a lower $32 \%$ relapse rate.

Although the study focused on the impact of antipsychotic maintenance on relapse, psychosocial factors such as expressed emotion also contribute to the risk of relapse. ${ }^{45}$ These factors must also be taken into consideration in clinical decision making for the individual patient. Use of illicit substances could also increase the risk of relapse. However, as the overall prevalence of substance misuse is much lower in Hong Kong than in some other populations, ${ }^{46}$ we did not do regular laboratory monitoring of illicit substance use. Finally, although quetiapine seems to be as effective as other antipsychotic drugs in the treatment of first episode psychosis, ${ }^{478}$ our design does not consider the question of the relative advantages or disadvantages of quetiapine compared with other antipsychotics as a maintenance treatment. Other antipsychotics could have different efficacies. Other atypical antipsychotics could be equally effective at a different cost, depending on regional pricing and availability of generic drugs. As a final consideration, this study specifically targeted a cohort of patients likely to have a better outcome, and for whom discontinuation of treatment was a realistic consideration. Although we did not recruit patients with residual psychotic symptoms, or those who already had at least one relapse, discontinuation in these patients would probably result in an even higher risk of relapse than found in this study. The low rate of substance misuse and high rate of employment in this group also need to be taken into consideration before generalising the findings to other settings.

\section{Conclusion and implications}

In summary, even patients with good response in positive symptoms and good functioning after a first episode of psychosis have a high risk of relapse, but this can be reduced with maintenance drug treatment. On the other hand, maintenance treatment with atypical antipsychotic drugs is still associated with appreciable side effects (table 5). This result highlights the challenges confronting clinicians and patients considering discontinuation of treatment after a single psychotic episode. These data provide a strong case for continuation of maintenance treatment to beyond one year after 
a first episode of psychosis, but we also acknowledge that the clinical decision for maintenance treatment must be made in an individualised manner taking into consideration factors such as side effects and the availability of effective monitoring for relapse. Currently identified predictors of relapse are still relatively non-specific, and further research is needed to inform decisions on maintenance or discontinuation for individual patients.

Contributions: EYHC, CLMH, MMLL, CWL, CPYC, SFH, and WGH conceived and designed the study. EYHC, CLMH, MMLL, CPYC, and DWSC obtained funding and supervised the study. EYHC, CLMH, MMLL, CPYC, CWL, DWSC, ST, EPFP, KTC, YCW, FYMM, KPMC, and SFH collected the data. CLMH, EYHC, WGH, and TJY analysed the data. All authors interpreted the data. EYHC, CLMH, and WGH drafted the manuscript. All authors had access to the data, critically revised the manuscript, and approved the final version. EYHC is the guarantor.

Funding: The study was supported by the Research Grants Council of Hong Kong (HKU 7655/05M) and an investigator initiated study award from AstraZeneca Pharmaceuticals. WGH was supported by the Michael Smith Foundation for Health Research and the British Columbia Mental Health and Addictions Services. AstraZeneca prepared the quetiapine and the placebo and packaged the study drugs according to the randomisation schedule. AstraZeneca played no role in the study design or conduct of the study; collection, management, analysis, and interpretation of the data; or preparation, review, and approval of the manuscript. The only study data provided to AstraZeneca were reports of serious adverse events. The authors' work was independent from the funders.

Competing interests: All authors have completed the Unified Competing Interest form at www.icmje.org/coi_disclosure.pdf (available on request from the corresponding author) and all authors declare (1) Financial support for the submitted work from Research Grants Council of Hong Kong and AstraZeneca Pharmaceuticals. (2) EYHC has participated in paid advisory boards for Otsuka, has received educational grant support from Janssen-Cilag, and has received research funding from AstraZeneca, Janssen-Cilag, Pfizer, Eli Lilly, Sanofi-Aventis, and Otsuka; MMLL has done consultancy for Otsuka and Eli Lilly; DWSC has received research grants from Janssen-Cilag and Pfizer; WGH has participated in advisory boards for In-Silico Biosciences, Wyeth/Solvay, Janssen-Cilag, and AstraZeneca; has done consultancy for Novartis and AstraZeneca; and has received lecture fees from Janssen-Cilag, Pfizer, and AstraZeneca and educational grant support from Janssen-Cilag, Eli Lilly, and AstraZeneca; CLMH, CPYC CWL, ST, EPFP, KTC, YCW, FYMM, KPMC, TJY, and SFH declare no relationships with commercial entities that might have an interest in the submitted work in the previous 3 years. All authors declare that they have (3) no spouses, partners, or children with financial relationships with commercial entities that may be relevant to the submitted work, and (4) no non-financial interests that may be relevant to the submitted work. Ethical approval: The institutional review boards at each site gave ethical approval, and all participants provided written informed consent. Data sharing: No additional data available.

1 Robinson DG, Woerner MG, Delman HM, Kane JM. Pharmacological treatments for first-episode schizophrenia. Schizophr Res 2005;31:705-22.

2 Lieberman JA, Jody D, Geisler S, Alvir J, Loebel A, Szymanski S, et al. Time course and biologic correlates of treatment response in firstepisode schizophrenia. Arch Gen Psychiatry 1993;50:369-76.

3 Robinson D, Woerner MG, Alvir JMJ, Bilder R, Goldman R, Geisler S, et al. Predictors of relapse following response from a first episode of schizophrenia or schizoaffective disorder. Arch Gen Psychiatry 1999;56:241-7.

4 Weiden PJ, Olfson M. Cost of relapse in schizophrenia. Schizophr Bull 1995;21:419-29.

5 Lieberman JA, Alvir JM, Koreen A, Geisler S, Chakos M, Sheitman B, et al. Psychobiologic correlates of treatment response in schizophrenia. Neuropsychopharmacology 1996;14(suppl 3):13-21S.

6 Gilbert PL, Harris MJ, McAdams LA, Jeste DV. Neuroleptic withdrawal in schizophrenic patients: a review of the literature. Arch Gen Psychiatry 1995;52:173-88.

7 Csernansky JG, Mahmoud R, Brenner R, for the Risperidone-USA-79 Study Group. A comparison of risperidone and haloperidol for the prevention of relapse in patients with schizophrenia. $N$ Engl J Med 2002;346:16-22.

8 Leucht S, Barnes TR, Kissling W, Engel RR, Correll C, Kane JM. Relapse prevention in schizophrenia with new-generation antipsychotics: a systematic review and exploratory meta-analysis of randomized, controlled trials. Am J Psychiatry 2003;160:1209-22.

9 Laruelle $M$. The role of endogenous sensitization in the pathophysiology of schizophrenia: implications from recent brain maging studies. Brain Res Rev 2000;31:371-84.

10 Crow TJ, MacMillan JF, Johnson AL, Johnstone EC. The Northwick Park study of first episodes of schizophrenia II: a randomized controlled trial of prophylactic neuroleptic treatment. Br J Psychiatry 1986;148:120-7.

11 Kane JM, Rifkin A, Quitkin F, Nayak D, Ramos-Lorenzi J. Fluphenazine vs placebo in patients with remitted, acute first-episode schizophrenia. Arch Gen Psychiatry 1982;39:70-3.

12 Hogarty GE, Ulrich RF. The limitations of antipsychotic medication on schizophrenia relapse and adjustment and the contributions of psychosocial treatment. J Psychiatry Res 1998;32:243-50.

13 American Psychiatric Association. Practice guideline for the treatment of patients with schizophrenia. 2nd ed. American Psychiatric Association, 2004

14 Royal Australian and New Zealand College of Psychiatrists Clinical Practice Guidelines Team for the Treatment of Schizophrenia and Related Disorders. Royal Australian and New Zealand College of Psychiatrists clinical practice guidelines for the treatment of schizophrenia and related disorders. Aust N Z J Psychiatry 2005;39:1-30

15 Kane JM, Leucht S. Unanswered questions in schizophrenia clinical trials. Schizophr Bull 2008;34:302-9.

16 Wyatt RJ. Risks of withdrawing antipsychotic medications. Arch Gen Psychiatry 1995;52:205-8.

17 Carpenter WT. Maintenance therapy of persons with schizophrenia. Clin Psychiatry 1996;57(suppl 9):10-8S.

18 Robinson DG, Woerner MG, Alvir JM, Bilder RM, Hinrichsen GA, Lieberman JA. Predictors of medication discontinuation by patients with first-episode schizophrenia and schizoaffective disorder. Schizophr Res 2002;57:209-19.

19 Gitlin M, Nuechterlein K, Subotnik KL, Ventura J, Mintz J, Fogelson DL, et al. Clinical outcome following neuroleptic discontinuation in patients with remitted recent-onset schizophrenia. Am J Psychiatry 2001;158:1835-42.

20 Wunderink L, Nienhuis F), Sytema S, Slooff CJ, Knegtering R, Wiersma D. Guided discontinuation versus maintenance treatment in remitted first-episode psychosis: relapse rates and functional outcome. J Clin Psychiatry 2007;68:654-61.

21 Kay SR, Fiszbein A, Opler LA. The positive and negative syndrome scale (PANSS) for schizophrenia. Schizophr Bull 1987;13:261-76.

22 Coldham EL, Addington J, Addington D. Medication adherence of individuals with a first episode of psychosis. Acta Psychiatr Scand 2002;106:286-90.

23 Bradford DW, Perkins DO, Lieberman JA. Pharmacological management of first-episode schizophrenia and related nonaffective psychoses. Drugs 2003;63:2265-83.

24 Chen E. Developing an early intervention service in Hong Kong. In: Ehmann T, MacEwan GW, Honer WG, eds. Best care in early psychosis intervention: global perspectives. Taylor and Francis, 2004:125-30.

25 American Psychiatric Association. Diagnostic and statistical manual of mental disorders: DSM-IV. 4th ed. American Psychiatric Association, 1994

26 So E, Kam I, Leung CM, Chung D, Liu Z, Fong S. The Chinese-bilingua SCID-I/P project: stage 1-reliability for mood disorders and schizophrenia. Hong Kong J Psychiatry 2003;13:7-18.

27 Guy W. Clinical global impression scale (CGI). In: Guy W, ed. ECDEU assessment manual for psychopharmacology. Revised ed. Dept of Health, Education and Welfare, National Institute of Mental Health, 1976:217-22.

28 Berger GE, Proffitt TM, McConchie M, Kerr M, Markelev C, Yuen HP, et al. Dosing quetiapine in drug-naive first-episode psychosis: a controlled, double-blind, randomized, single-center study investigating efficacy, tolerability, and safety of $200 \mathrm{mg} /$ day vs $400 \mathrm{mg} /$ day of quetiapine fumarate in 141 patients aged 15 to 25 years. J Clin Psychiatry 2008;69:1702-14.

29 Vaughn CE, Snyder KS, Jones S, Freeman WB, Falloon IR. Family factors in schizophrenic relapse: replication in California of British research on expressed emotion. Arch Gen Psychiatry 1984;41:1169-77.

30 Goldman HH, Skodol AE, Lave TR. Revising axis V for DSM-IV: a review of measures of social functioning. Am J Psychiatry 1992;149:1148-56.

31 Cannon-Spoor HE, Potkin SG, Wyatt RJ. Measurement of premorbid adjustment in chronic schizophrenia. Schizophr Bull 1982;8:470-84 
32 Foerster A, Lewis S, Owen M, Murray R. Pre-morbid adjustment and personality in psychosis: effects of sex and diagnosis. Br J Psychiatry 1991;158:171-6.

33 Andreasen NC. The scale for the assessment of negative symptoms (SANS). University of lowa, 1983.

34 Andreasen NC. The scale for the assessment of positive symptoms (SAPS). University of lowa, 1984.

35 Amador XF, Flaum M, Andreasen NC, Strauss DH, Yale SA, Clark S, et al. Awareness of illness in schizophrenia and schizoaffective and mood disorders. Arch Gen Psychiatry 1994;51:826-36.

36 Lingjaerde O, Ahlfors UG, Bech P, Dencker SJ, Elgen K. The UKU side effect rating scale: a new comprehensive rating scale for psychotropic drugs and a cross-sectional study of side effects in neuroleptic-treated patients. Acta Psychiatr Scand 1987;76(suppl 334):1-100S.

37 Barnes TR. A rating scale for drug-induced akathisia. Br J Psychiatry 1989;154:672-6.

38 Guy W. Abnormal involuntary movements scale (AIMS). In: Guy W, ed. ECDEU assessment manual for psychopharmacology. Revised ed. Dept of Health, Education and Welfare, National Institute of Mental Health, 1976:534-7.

39 Simpson GM, Angus JW. A rating scale for extrapyramidal side effects. Acta Psychiatr Scand 1970;45(suppl 212):11-9S

40 Collett $D$. Modelling survival data in medical research. 1st ed. Chapman and Hall, 1994.

41 Hollis S, Campbell F. What is meant by intention to treat analysis? Survey of published randomised controlled trials. BMJ 1999;319:670-4.
42 Clark TG, Bradburn MJ, Love SB, Altman DG. Survival analysis part IV: further concepts and methods in survival analysis. Br J Cancer 2003;89:781-6.

43 Lin D, Wei L, Ying Z. Checking the Cox model with cumulative sums of martingale-based residuals. Biometrika 1993;80:557-72.

44 Lieberman JA, Stroup TS, McEvoy JP, Swartz MS, Rosenheck RA, Perkins DO, et al. Effectiveness of antipsychotic drugs in patients with chronic schizophrenia. N Engl J Med 2005;353:1209-23.

45 Park G, Hadzi-Pavlovic, D. Expressed emotion as a predictor of schizophrenic relapse: an analysis of aggregated data. Psychol Med 1990;20:961-5.

46 Lau JTF, Kim KH, Tsui HY. Prevalence, health outcomes, and patterns of psychotropic substance use in a Chinese population in Hong Kong: a population-based study. Subst Use Misuse 2005;40:187-209.

47 McEvoy JP, Lieberman JA, Perkins DO, Hamer RM, Gu H, Lazarus A, et al. Efficacy and tolerability of olanzapine, quetiapine, and risperidone in the treatment of early psychosis: a randomized, double-blind 52-week comparison. Am J Psychiatry 2007;164:1050-60.

48 Kahn RS, Fleischhacker WW, Boter H, Davidson M, Vergouwe Y, Keet IPM, et al. Effectiveness of antipsychotic drugs in first-episode schizophrenia and schizophreniform disorder: an open randomised clinical trial. Lancet 2008;371:1085-97.

Accepted: 7 June 2010 